\title{
PERIODS OF MODULAR FORMS AND IDENTITIES BETWEEN EISENSTEIN SERIES
}

\author{
KAMAL KHURI-MAKDISI AND WISSAM RAJI
}

\begin{abstract}
Borisov and Gunnells observed in 2001 that certain linear relations between products of two holomorphic weight 1 Eisenstein series had the same structure as the relations between periods of modular forms; a similar phenomenon exists in higher weights. We give a conceptual reason for this observation in arbitrary weight. This involves an unconventional way of expanding the Rankin-Selberg convolution of a cusp form with an Eisenstein series. We also prove a partial result towards understanding the action of a Hecke operator on a product of two Eisenstein series.
\end{abstract}

\section{INTRODUCTION}

Let $N \geq 3$. In a series of articles BG01a, BG01b, BG03, L. Borisov and P. Gunnells introduced the concept of a toric modular form on $\Gamma_{1}(N)$, defined in terms of lattices and polytopes. They proved that toric modular forms were polynomials in certain Eisenstein series of weight 1, and identified the cuspidal part of the algebra generated by these polynomials in weight 1 Eisenstein series: the cuspidal part contained everything in weights $\geq 3$, and contained precisely the span of Hecke eigenforms in weight 2 with nontrivial central $L$-value.

In [KM12, the first named author of this article investigated a different way of producing modular forms on $\Gamma(N)$ coming from Laurent expansions of elliptic functions and the moduli problem of elliptic curves with $N$-torsion, and showed that this also led to a ring of modular forms generated by Eisenstein series of weight 1 . In full level $\Gamma(N)$, this ring contains every modular form of weight 2 and above.

In both approaches, a key fact underlying the proofs is an identity between products of pairs of Eisenstein series. For weight 1 Eisenstein series, this identity states that if $\lambda+\mu+\nu=0$, then $E_{1, \lambda} E_{1, \mu}+E_{1, \mu} E_{1, \nu}+E_{1, \nu} E_{1, \lambda} \in \mathcal{E}_{2}$, the space of weight 2 Eisenstein series. (The precise definition of the Eisenstein series is given below, in (1.1) and (1.2).) By Remark 3.12 of [BG01b, this relation is parallel to Manin's three-term relation (see (1.7) below) between modular symbols. For Eisenstein series of higher weight, the analogous result is in Section 6 of [BG03].

The parallelism between Manin's relations on modular symbols on one hand, and linear relations between products of pairs of Eisenstein series on the other hand, was extended and codified by Paşol in Paş06. But this parallelism still remained an experimental observation, despite the feeling that it was not just coincidental.

In this article, we explain this parallelism conceptually, based on an interesting expression that we derive in Proposition 2.5 for the Petersson inner product

2000 Mathematics Subject Classification. 11F67, 11F11.

Key words and phrases. Modular forms, periods, Eisenstein series.

January 17, 2016. 
$\left\langle f, E_{\ell, \lambda} E_{m, \mu}\right\rangle$ between a cusp form $f$ of weight $k=\ell+m$, and a product of two Eisenstein series. Our result expresses the inner product in terms of pairings between $f$ and certain modular symbols (i.e., in terms of periods of $f$ ). This makes the connection with relations between modular symbols transparent, and gives a direct way to understand the above parallelism, which we describe in Theorem 2.8 the resulting identity, however, can include nonholomorphic Eisenstein series, due to the presence of terms like $E_{2, \lambda}$ in various products. In Section 3 , we translate the identity of Theorem 2.8 to the setting of holomorphic Eisenstein series, so that every Eisenstein series of weight 2 appears as a difference, as in $E_{2, \lambda}-E_{2, \mu}$. We also make the connection with the results of Paş06 and with classical identities involving elliptic functions. Finally, in Section 4 we include a partial result related to the Hecke action; Section 7 of [BG01b, BG03] in fact shows that their homomorphism from the space of modular symbols to the cuspidal space of toric modular forms respects the Hecke action (the fact that the homomorphism is well-defined in the first place is due to the parallelism with the Manin relations). We do not know whether the methods there extend to $\Gamma(N)$. What we are able to accomplish in this article is to follow a proof in Section 4 of KM12 that yields good behavior of only certain traces from higher level $\Gamma(N M)$ to $\Gamma(N)$. That result, combined with a certain "subtle symmetry" in the space of weight 1 Eisenstein series on $\Gamma(N)$, was sufficient to deduce the result for all traces in weights 2 and 3 in KM12, but would need an extra idea in order to work for higher weights.

It is reasonable to expect that $\left\langle f, E_{\ell, \lambda} E_{m, \mu}\right\rangle$ should be related to periods of $f$. For instance, if $f$ is a newform of weight $k$, then as is well known from Theorem 2 and equation (4.3) of Shi76], for many choices of Dirichlet characters $\chi_{1}, \chi_{2}$ and integers $\ell+m=k$, it is possible to find suitable Eisenstein series $G_{\ell} \in \mathcal{E}_{\ell}, G_{m} \in \mathcal{E}_{m}$ for which $\left\langle f, G_{\ell} G_{m}\right\rangle=L\left(\ell, f \otimes \chi_{1}\right) L\left(m, f \otimes \chi_{2}\right)$. This is the usual method of RankinSelberg, which involves unfolding the integral along the sum of one Eisenstein series, while using the Fourier $(q-)$ expansions of $f$ and the second Eisenstein series. Moreover, the special values of the twisted $L$-function of $f$ can be expressed as periods. However, there is no way for such an identity to hold for arbitrary $f$ that is not a Hecke eigenform: most notably, the inner product $\left\langle f, G_{\ell} G_{m}\right\rangle$ is linear in $f$, while the product $L\left(\ell, f \otimes \chi_{1}\right) L\left(m, f \otimes \chi_{2}\right)$ of special values is quadratic in $f$. Instead, we unfold the inner product along the simultaneous sums of both Eisenstein series, and obtain an infinite sum of integrals of the form $\int_{\mathcal{H}} f(z)(a z+b)^{-\ell}(c z+d)^{-m} y^{k} d_{\mathcal{H}} z$. These integrals have already been related to periods of $f$, by work of $H$. Cohen as cited in pp. 204-205 of KZ84. In our setting, we actually need to evaluate the result after replacing $(a z+b)^{-\ell}$ by $(a z+b)^{-\ell}|a z+b|^{-s}$, and similarly for $(c z+d)^{-m}$; this is due to convergence issues with the Eisenstein series. We do not believe that the integral has been carried out in precisely this form before, and hope that the computation will be of independent interest.

We speculate that the parallelism between the structure of the Manin relations and the structure of some relations between Eisenstein series might lead to a different way to produce spaces of modular forms, once a space of modular symbols has been computed.

Notation. In the rest of the introduction, we fix notation and recall various standard facts.

We first recall the definition of the Eisenstein series on $\Gamma=\Gamma(N)$. This works for any level $N \geq 1$, although at various places we assume $N \geq 3$, to avoid the 
minor inconvenience of having $\left(\begin{array}{cc}-1 & 0 \\ 0 & -1\end{array}\right)$ belong to $\Gamma$. The Eisenstein series will be parametrized by an element $\lambda=\left(\lambda_{1}, \lambda_{2}\right) \in N^{-1} \mathbf{Z}^{2}$; in fact, only the image of $\lambda$ in $\mathbf{Q}^{2} / \mathbf{Z}^{2}$ matters, but the level definitely depends on the denominator of $\lambda$. We then define as usual an Eisenstein series of weight $\ell \geq 1$ by

$$
E_{\ell, \lambda}(z, s)=\sum_{\substack{(a, b) \equiv \lambda\left(\bmod \mathbf{Z}^{2}\right) \\(a, b) \neq(0,0)}}(a z+b)^{-\ell}|a z+b|^{-s} y^{s / 2} .
$$

As usual, $z=x+i y \in \mathcal{H}$, the complex upper half plane. The series in (1.1) converges absolutely for $\operatorname{Re} s+\ell>2$, and has an analytic continuation for all $s \in \mathbf{C}$. It is well known that, in fact, $\Gamma((s / 2)+\ell) E_{\ell, \lambda}(z, s)$ is an entire function of $s$; see for example Theorem 9.7 of [Shi07] (note that $s$ there corresponds to $s / 2$ here). We write

$$
E_{\ell, \lambda}(z)=E_{\ell, \lambda}(z, 0) \text {. }
$$

The Eisenstein series of (1.2) are holomorphic functions of $z$ except when $\ell=2$, in which case they have the form $E_{2, \lambda}(z)=-\pi y^{-1}+$ holomorphic function. Thus $E_{2, \lambda}-E_{2, \mu}$ is actually a holomorphic function of $z$. In general, we have used the notation $\mathcal{E}_{\ell}$ above to refer to the space of (holomorphic) forms in the span of all $E_{\ell, \lambda}$, as $\lambda$ varies in $N^{-1} \mathbf{Z}^{2}$; the holomorphy is automatic except in weight 2.

For precision, we normalize the action of an element $\gamma=\left(\begin{array}{ll}a & b \\ c & d\end{array}\right) \in G L(2, \mathbf{R})^{+}$on a function $f: \mathcal{H} \rightarrow \mathbf{C}$ so that it factors through $P G L(2)$, or more accurately through $G L(2, \mathbf{R})^{+} / Z^{0}$, where $Z^{0} \cong \mathbf{R}^{+*}$ is the connected component of the identity in the center $Z$ of $G L(2, \mathbf{R})$ :

$$
\left(\left.f\right|_{k} \gamma\right)(z)=f(\gamma z)(c z+d)^{-k}(\operatorname{det} \gamma)^{k / 2}
$$

Thus, for example, an element $f \in \mathcal{S}_{k}$, the space of holomorphic cusp forms of weight $k$ and level $N$, will satisfy $\left.f\right|_{k} \gamma=f$ for all $\gamma \in \Gamma(N)$; similarly for $\mathcal{E}_{k}$. However, we will frequently need to use this notation for all sorts of functions $f$ on $\mathcal{H}$, and the matrix $\gamma$ can have any positive determinant in many occasions below.

We also distinguish notation for various pairings. For $f, g$ transforming with the same weight $k$ under a congruence subgroup $\Gamma$, the Petersson inner product is

$$
\langle f, g\rangle_{\Gamma}=\int_{z \in \mathcal{F}} f(z) \overline{g(z)} y^{k} d_{\mathcal{H}} z
$$

whenever the integral converges, where $\mathcal{F}$ is a fundamental domain for $\Gamma \backslash \mathcal{H}$, and the $G L(2, \mathbf{R})^{+}$-invariant measure on $\mathcal{H}$ is as usual $d_{\mathcal{H}} z=y^{-2} d x d y$, with $z=x+i y$. As usual, the spaces $\mathcal{S}_{k}$ and $\mathcal{E}_{k}$ are orthogonal with respect to the Petersson inner product.

In the above definition, unlike the beginning of the introduction, we have included a subscript $\Gamma$ in the Petersson inner product. This is so as to accentuate the difference between the Petersson inner product and the full integral over $\mathcal{H}$, which makes several appearances in this article:

$$
\langle f, g\rangle_{\mathcal{H}}=\int_{z \in \mathcal{H}} f(z) \overline{g(z)} y^{k} d_{\mathcal{H}} z .
$$

Finally, we define the period pairing for $f \in \mathcal{S}_{k}$ and $P(z)$ a polynomial of degree $\leq w$, where we always write $w=k-2$ :

$$
[f(z), P(z)]=\int_{z=0}^{i \infty} f(z) P(z) d z .
$$


The Manin relations, which follow from the Cauchy integral theorem, state that

$$
[f, P]+\left[\left.f\right|_{k} \sigma,\left.P\right|_{-w} \sigma\right]=0, \quad[f, P]+\left[\left.f\right|_{k} \tau,\left.P\right|_{-w} \tau\right]+\left[\left.f\right|_{k} \tau^{2},\left.P\right|_{-w} \tau^{2}\right]=0
$$

where

$$
\sigma=\left(\begin{array}{cc}
0 & -1 \\
1 & 0
\end{array}\right), \quad \tau=\left(\begin{array}{cc}
0 & 1 \\
-1 & -1
\end{array}\right) .
$$

The Manin relations are usually stated in terms of an identity between symbols, as in Mer94 for example, but we will only need the period pairing with cusp forms in this article.

\section{MAin COMPUtATion}

Let $\ell, m \geq 1$ such that $\ell+m=k$. Take $\lambda, \mu \in N^{-1} \mathbf{Z}^{2}$, and take a weight $k$ cusp form $f \in \mathcal{S}_{k}(\Gamma)$ where we fix the notation $\Gamma=\Gamma(N)$. Our goal in this section is to relate the Petersson inner product $\left\langle f(z), E_{\ell, \lambda}(z, \bar{p}) E_{m, \mu}(z, \bar{q})\right\rangle_{\Gamma}$ to periods of $f$. As a first step, we study the integrals that arise when we expand the Eisenstein series.

Definition 2.1. Let $\ell, m, k, p, q$ be as above (in particular, $\ell+m=k$ ), and take a cusp form $f \in \mathcal{S}_{k}$. Let $a, b, c, d \in \mathbf{Z}$ and write $M=\left(\begin{array}{ll}a & b \\ c & d\end{array}\right)$. (The matrix $M$ is allowed to be singular.) Let $S \subset \mathcal{H}$ be a measurable subset - typically, $S$ is either $\mathcal{H}$ or a fundamental domain for a congruence subgroup. We then define

$$
\begin{aligned}
& I_{M, S, f, \ell, m, p, q, k}=I_{M, S, f} \\
& \quad=\int_{z \in S} f(z)(a \bar{z}+b)^{-\ell}|a z+b|^{-p}(c \bar{z}+d)^{-m}|c z+d|^{-q} y^{p / 2+q / 2+k} d_{\mathcal{H}} z,
\end{aligned}
$$

whenever the integral converges absolutely.

Two particular values of the matrix $M$ will play a prominent role in what follows:

$$
\mathcal{I}=\left(\begin{array}{ll}
1 & 0 \\
0 & 1
\end{array}\right), \quad \mathcal{N}=\left(\begin{array}{ll}
0 & 1 \\
1 & 0
\end{array}\right)
$$

The names stand for "identity" and "negative determinant", respectively.

Proposition 2.2. The integrals $I_{M, S, f}$ have the following properties:

(1) For $\gamma \in G L(2, \mathbf{R})^{+}$, we have

$$
I_{M \gamma, S, f}=(\operatorname{det} \gamma)^{-(p+q+k) / 2} I_{M, \gamma S,\left.f\right|_{k} \gamma^{-1}} .
$$

(2) If $M=\mathcal{I}$, then

$$
I_{\mathcal{I}, \mathcal{H}, f}=i^{\ell} \pi 2^{-k-p / 2-q / 2+2} \frac{\Gamma(k+p / 2+q / 2-1)}{\Gamma(p / 2+\ell) \Gamma(q / 2+m)} \int_{y=0}^{\infty} f(i y) y^{m-1-p / 2+q / 2} d y .
$$

In the special case $p=q=s$, this yields

$$
I_{\mathcal{I}, \mathcal{H}, f}=i^{k-2} \pi 2^{-k-s+2} \frac{\Gamma(k+s-1)}{\Gamma(s / 2+\ell) \Gamma(s / 2+m)}\left[f,(-z)^{m-1}\right] .
$$

(3) If $M=\mathcal{N}$, then

$$
I_{\mathcal{N}, \mathcal{H}, f}=i^{m} \pi 2^{-k-p / 2-q / 2+2} \frac{\Gamma(k+p / 2+q / 2-1)}{\Gamma(p / 2+\ell) \Gamma(q / 2+m)} \int_{y=0}^{\infty} f(i y) y^{\ell-1+p / 2-q / 2} d y .
$$


In the special case $p=q=s$, this yields

$$
I_{\mathcal{N}, \mathcal{H}, f}=i^{k-2} \pi 2^{-k-s+2} \frac{\Gamma(k+s-1)}{\Gamma(s / 2+\ell) \Gamma(s / 2+m)}\left[f,(-z)^{\ell-1}\right] .
$$

Proof. Statement (1) is straightforward, and statements (2) and (3) are equivalent. We therefore prove statement (2). Due to analytic continuation, it suffices to prove identity (2.4) under the assumption that $\operatorname{Re} q \gg \operatorname{Re} p \gg 0$. Let us first show that the integral in $I_{\mathcal{I}, \mathcal{H}, f}$ converges absolutely under this assumption; later on, in Remark 2.3 we will in fact show that we have absolute convergence under the weaker condition $\operatorname{Re} p, \operatorname{Re} q \gg 0$. This will imply that (2.5) is meaningful for Re $s \gg 0$, and will complete the proof.

To show that $I_{\mathcal{I}, \mathcal{H}, f}$ converges absolutely for $\operatorname{Re} q \gg \operatorname{Re} p \gg 0$, note that, uniformly in $x, y^{k / 2}|f(z)|$ is bounded as $y \rightarrow 0$ and decreases exponentially as $y \rightarrow \infty$. Integrating the absolute value first over $x$ (with the substitution $x=y \xi$ ) and then over $y$ shows that sufficient conditions for convergence are $\operatorname{Re} p+\ell>1$ and $\operatorname{Re} q-\operatorname{Re} p+k-2 \ell>0$. The right hand side of (2.4), on the other hand, is holomorphic for $2 k+\operatorname{Re} p+\operatorname{Re} q-2>0$, since the Mellin transform of $f$ is entire, as usual, using exponential decay of $f(i y)$ as $y \rightarrow 0$ through real values of $y$.

We can now evaluate the original integral for $I_{\mathcal{I}, \mathcal{H}, f}$. Make again the substitution $x=y \xi$, so $z=i y(1-i \xi)$ and $d_{\mathcal{H}} z=y^{-1} d y d \xi$. This yields

$$
I_{\mathcal{I}, \mathcal{H}, f}=i^{\ell} \int_{\xi \in \mathbf{R}}(1+i \xi)^{-\ell}|1+i \xi|^{-p} \int_{y=0}^{\infty} f(i y(1-i \xi)) y^{-p / 2+q / 2+m-1} d y d \xi
$$

In the inner integral over $y$, we can substitute $u=y(1-i \xi)$ and shift the contour of $u$ so that $u$ goes from 0 to $+\infty$ along real values. (This uses the estimates on $y^{k / 2}|f(z)|$ mentioned above, as well as the assumption $\operatorname{Re} q \gg \operatorname{Re} p$. A related alternative way is to expand $f(z)=\sum_{n \geq 1} c_{n} e^{i n H z}$ for some $H$ depending on the width of the cusp at $\infty$, bearing in mind that the $c_{n}$ grow at worst like a power of n.) Our desired result (2.4) then boils down to evaluating

$$
\int_{\xi \in \mathbf{R}}(1+i \xi)^{-\alpha}(1-i \xi)^{-\beta} d \xi, \quad \text { where } \alpha=p / 2+\ell, \quad \beta=q / 2+m .
$$

This (standard) integral can be evaluated for $\operatorname{Re} p, \operatorname{Re} q \gg 0$ by considering the following function and its Fourier transform $\hat{h}(u)=\int_{t \in \mathbf{R}} h(t) \exp (-2 \pi i t u) d t$ :

$$
h_{b}(t)=\left\{\begin{array}{ll}
e^{-2 \pi t} t^{b-1} & \text { if } t>0, \\
0 & \text { otherwise, }
\end{array} \quad \hat{h}_{b}(u)=(2 \pi(1+i u))^{-b} \Gamma(b),\right.
$$

as well as the fact that the Fourier transform preserves the $L^{2}$ inner product:

$$
\int_{t \in \mathbf{R}} h_{\alpha}(t) \overline{h_{\bar{\beta}}(t)} d t=\int_{u \in \mathbf{R}} \hat{h}_{\alpha}(u) \overline{\hat{h}_{\bar{\beta}}(u)} d u .
$$

Putting all this together completes the proof, except for the comment on convergence for $\operatorname{Re} p, \operatorname{Re} q \gg 0$, which we deal with in the following remark.

Remark 2.3. In this remark, we show by a more careful analysis that the integral in $I_{\mathcal{I}, \mathcal{H}, f}$ converges absolutely when $\operatorname{Re} p+\ell, \operatorname{Re} q+m>2$. This will follow, as we shall see, from the assertion that the inner product $\left\langle f(z), E_{\ell, \lambda}(z, \bar{p}) E_{m, \mu}(z, \bar{q})\right\rangle_{\Gamma}$ converges absolutely in this range of $(p, q)$, even after one replaces the sums in the Eisenstein series $E_{\ell, \lambda}, E_{m, \mu}$ by sums over the absolute values of their terms. 
This good convergence of the integral of the sums will justify all our subsequent manipulations in Proposition 2.5.

Let us first explain why good behavior of $\left\langle f, E_{\ell, \lambda} E_{m, \mu}\right\rangle_{\Gamma}$ implies the same for $I_{\mathcal{I}, \mathcal{H}, f}$. Let $\mathcal{F} \subset \mathcal{H}$ be a fundamental domain for $\Gamma=\Gamma(N)$. (The following implicitly assumes that $N \geq 3$, so that $\left(\begin{array}{cc}-1 & 0 \\ 0 & -1\end{array}\right) \notin \Gamma$; otherwise, a minor change fixes the proof.) We can decompose the integral for $I$ into $I_{\mathcal{I}, \mathcal{H}, f}=\sum_{\gamma \in \Gamma(N)} I_{\mathcal{I}, \gamma \mathcal{F}, f}=$ $\sum_{\gamma} I_{\gamma, \mathcal{F}, f}$, where we used (2.3) in the last step, as well as the fact that $f$ transforms under $\Gamma(N)$. Putting absolute values into all the sums gives the following expression as a bound for $I_{\mathcal{I}, \mathcal{H}, f}$ :

$$
\int_{z \in \mathcal{F}}|f(z)| \sum_{\gamma=\left(\begin{array}{ll}
a & b \\
c & d
\end{array}\right)}|a z+b|^{-\ell-\operatorname{Re} p}|c z+d|^{-m-\operatorname{Re} q} y^{\operatorname{Re} p / 2+\operatorname{Re} q / 2+k} d_{\mathcal{H}} z .
$$

In this bound, the choice of $\gamma=\left(\begin{array}{ll}a & b \\ c & d\end{array}\right) \in \Gamma(N)$ in the sum runs over a proper subset of all $\left\{(a, b, c, d) \in \mathbf{Z}^{4} \mid(a, b) \neq(0,0) \neq(c, d)\right\}$, so we see that we can (wastefully) bound this by including all the other $(a, b, c, d)$ terms in the sum. This yields (an absolute value version of) the integral of $f$ against the product $E_{\ell, 0} E_{m, 0}$. For the application to Proposition 2.5 we will show convergence more generally for the integral of $f$ against $E_{\ell, \lambda} E_{m, \mu}$.

We thus want to study convergence of the expression (2.12) when $\left(\begin{array}{ll}a & b \\ c & d\end{array}\right)$ ranges over the larger set $X_{\lambda, \mu}$ of elements where $(a, b) \equiv \lambda \bmod \mathbf{Z}^{2},(c, d) \equiv \mu \bmod \mathbf{Z}^{2}$, and $(a, b) \neq(0,0) \neq(c, d)$ (see (2.13) below). We note that the fundamental domain $\mathcal{F}$ is contained in a finite union of translates (under $G L(2, \mathbf{Q})^{+}$) of Siegel domains of the form $S_{C}=\left\{z|| x \mid \leq C, y \geq C^{-1}\right\}$ for some $C>0$. Using (2.3), we see that it is sufficient to show good convergence of (2.12), with $\mathcal{F}$ replaced by $S_{C}$, where for each translate we replace both the cusp form $f$ and the Eisenstein series by translates, so $f, \lambda, \mu$ may be slightly different for each of these finitely many integrals over $S_{C}$. However, it is standard that for $z \in S_{C}$, there exists a constant $K$ (depending only on $C$, and independent of $z$ or $(a, b)$ ) for which $|a z+b| \geq K|a i+b|$; a similar remark holds for $(c, d)$. Hence the above integral can be compared to the product of $\sum_{\left(\begin{array}{ll}a & b \\ c & d\end{array}\right) \in X_{\lambda, \mu}}|a i+b|^{-\ell-\operatorname{Re} p}|c i+d|^{-m-\operatorname{Re} q}$ with the integral $\int_{z \in S_{C}}|f(z)| y^{\operatorname{Re} p / 2+\operatorname{Re} q / 2+k} d_{\mathcal{H}} z$. The sum converges as usual as soon as $\operatorname{Re} p+\ell, \operatorname{Re} q+m>2$, and the integral converges because $f$ is a cusp form.

Definition 2.4. Let $\lambda=\left(\lambda_{1}, \lambda_{2}\right), \mu=\left(\mu_{1}, \mu_{2}\right) \in N^{-1} \mathbf{Z}^{2}$. We define the following sets of matrices:

$$
\begin{aligned}
X_{\lambda, \mu}= & \left\{M=\left(\begin{array}{ll}
a & b \\
c & d
\end{array}\right) \in M_{2}(\mathbf{Q}) \mid\right. \\
& \left.(a, b) \equiv \lambda \bmod \mathbf{Z}^{2},(c, d) \equiv \mu \bmod \mathbf{Z}^{2},(a, b) \neq(0,0) \neq(c, d)\right\} \\
X_{\lambda, \mu}^{+}= & \left\{M \in X_{\lambda, \mu} \mid \operatorname{det} M>0\right\} \\
X_{\lambda, \mu}^{-}= & \left\{M \in X_{\lambda, \mu} \mid \operatorname{det} M<0\right\} \\
X_{\lambda, \mu}^{0}= & \left\{M \in X_{\lambda, \mu} \mid \operatorname{det} M=0\right\}
\end{aligned}
$$

We will occasionally write $X_{\lambda, \mu}^{\bullet}$ to refer to any of the above, where $\bullet$ can be the empty string or one of,,+- 0 . The group $\Gamma=\Gamma(N)$ acts on any of these sets by right multiplication: if $M \in X_{\lambda, \mu}^{\bullet}$ and $\gamma \in \Gamma$, then $M \gamma \in X_{\lambda, \mu}^{\bullet}$. We also denote

$$
Y_{\lambda, \mu}^{\bullet}=X_{\lambda, \mu}^{\bullet} / \Gamma=\text { any set of representatives for the } \Gamma \text {-orbits on } X_{\lambda, \mu}^{\bullet} \text {. }
$$


Note that the sets $X_{\lambda, \mu}^{0}$ and $Y_{\lambda, \mu}^{0}$ may be empty. Roughly speaking, nonemptiness requires $\lambda$ and $\mu$ to be parallel vectors modulo $\mathbf{Z}^{2}$.

Proposition 2.5. Let $\ell, m, k, p, q, f$ be as above, and write $\ell=1+\ell^{\prime}, m=1+m^{\prime}$ so that $\ell^{\prime}+m^{\prime}=w=k-2$. Assume that the level $N$ satisfies $N \geq 3$ (this is minor; otherwise, one needs an extra factor of 2 arising from the presence of $-\mathcal{I} \in \Gamma$ ). Then the following identity holds for $\operatorname{Re} p+\ell, \operatorname{Re} q+m>2$, and each integral $I_{M^{\prime}, \mathcal{H}, f^{\prime}}$ in the sum below converges absolutely, as does the sum itself:

$$
\begin{aligned}
& \left\langle f(z), E_{\ell, \lambda}(z, \bar{p}) E_{m, \mu}(z, \bar{q})\right\rangle_{\Gamma} \\
& =\sum_{M \in Y_{\lambda, \mu}^{+}}(\operatorname{det} M)^{-(p+q+k) / 2} I_{\mathcal{I}, \mathcal{H},\left.f\right|_{k} M^{-1}}+\sum_{M \in Y_{\mu, \lambda}^{+}}(\operatorname{det} M)^{-(p+q+k) / 2} I_{\mathcal{N}, \mathcal{H},\left.f\right|_{k} M^{-1} .}
\end{aligned}
$$

In case $p=q=s$ with $\operatorname{Re} s+\min (\ell, m)>2$, the value of this expression is

$$
\begin{aligned}
& \left\langle f(z), E_{\ell, \lambda}(z, \bar{s}) E_{m, \mu}(z, \bar{s})\right\rangle_{\Gamma}=i^{w} \pi 2^{-w-s} G_{w, \ell^{\prime}, m^{\prime}}(s) \times \\
& \times\left[\sum_{M \in Y_{\lambda, \mu}^{+}}(\operatorname{det} M)^{-s-k / 2}\left[\left.f\right|_{k} M^{-1},(-z)^{m^{\prime}}\right]+\sum_{M \in Y_{\mu, \lambda}^{+}}(\operatorname{det} M)^{-s-k / 2}\left[\left.f\right|_{k} M^{-1},(-z)^{\ell^{\prime}}\right]\right] .
\end{aligned}
$$

where

$$
G_{w, \ell^{\prime}, m^{\prime}}(s)=\frac{\Gamma(s+w+1)}{\Gamma\left(s / 2+\ell^{\prime}+1\right) \Gamma\left(s / 2+m^{\prime}+1\right)} .
$$

Note for future use that $G_{w, \ell^{\prime}, m^{\prime}}(0)=\left(\begin{array}{c}w \\ \ell^{\prime}\end{array}\right)=\left(\begin{array}{c}w \\ m^{\prime}\end{array}\right)$.

Proof. The assertions regarding convergence follow from redoing the proof that we are about to present with absolute values everywhere, and invoking the arguments in Remark 2.3. We leave the details to the reader, and proceed with the actual computation. This uses a standard unfolding argument to evaluate the inner product. Let $\mathcal{F}$ be a fundamental domain for $\Gamma$. From the disjoint union $X_{\lambda, \mu}=X_{\lambda, \mu}^{+} \cup X_{\lambda, \mu}^{-} \cup X_{\lambda, \mu}^{0}$, we obtain

$$
\left\langle f(z), E_{\ell, \lambda}(z, \bar{p}) E_{m, \mu}(z, \bar{q})\right\rangle_{\Gamma}=T^{+}+T^{-}+T^{0}, \quad T^{\bullet}=\sum_{M \in X_{\lambda, \mu}^{\bullet}} I_{M, \mathcal{F}, f} .
$$

For any $M$ in the above sum, write $\Gamma_{M}=\{\gamma \in \Gamma \mid M \gamma=M\}$ for the stabilizer of $M$. If $M \in X^{+} \cup X^{-}$, then $\Gamma_{M}=\{\mathcal{I}\}$, but elements of $X^{0}$ have nontrivial stabilizers that are parabolic subgroups. Write $\mathcal{F}_{M}$ for a fundamental domain of $\Gamma_{M} \backslash \mathcal{H}$. Then, using part (1) of Proposition 2.2, the invariance of $f$ under $\Gamma$, and the fact that $I_{M, S, f}$ is countably additive in $S$, we obtain as usual

$$
\begin{aligned}
T^{\bullet} & =\sum_{M \in Y_{\dot{\lambda}, \mu}} I_{M, \mathcal{F}_{M}, f} & & (\text { for } \bullet \in\{+,-, 0\}) \\
& =\sum_{M \in Y_{\dot{\lambda}, \mu}} I_{M, \mathcal{H}, f} & & (\text { only for } \bullet \in\{+,-\}) .
\end{aligned}
$$

From the above and another use of Proposition 2.2(1), we obtain that $T^{+}$is equal to the first term on the right hand side of (2.15). With a little more work, we obtain that $T^{-}$is equal to the second term on the right hand side of (2.15); the 
point is that we have a bijection $M \in X_{\mu, \lambda}^{+} \mapsto \mathcal{N} M \in X_{\lambda, \mu}^{-}$that descends to a similar bijection from $Y_{\mu, \lambda}^{+}$to $Y_{\lambda, \mu}^{-}$.

It remains to show that $T^{0}=0$. In principle, this can be shown directly by showing that the sum of the terms coming from singular $M$ in the product $E_{\ell, \lambda}(z, \bar{p}) E_{m, \mu}(z, \bar{q})$ is an Eisenstein series; indeed, $\operatorname{det} M=0$ occurs precisely when $(c, d)=(\kappa a, \kappa b)$ for some $\kappa \neq 0$, and in that case we essentially obtain a sum over $(a, b)$ of $(a \bar{z}+b)^{-\ell-m}|a z+b|^{-p-q}$, including some annoyance from factors involving $\kappa$. We prefer to argue instead using the stabilizer $\Gamma_{M}$, in the style of "negligible orbits" appearing in the Rankin-Selberg method on higher rank groups.

For $M \in Y_{\lambda, \mu}^{0}$, we have $\operatorname{det} M=0$; hovever, both rows of $M$ are nonzero, hence their span is a one-dimensional $\mathbf{Q}$-rational subspace of $\mathbf{Q}^{2}$. This subspace contains a primitive integral vector $\left(c^{\prime}, d^{\prime}\right) \in \mathbf{Z}^{2}$ with $\operatorname{gcd}\left(c^{\prime}, d^{\prime}\right)=1$. Choose $a^{\prime}, b^{\prime} \in \mathbf{Z}$ so that $\gamma:=\left(\begin{array}{cc}a^{\prime} & b^{\prime} \\ c^{\prime} & d^{\prime}\end{array}\right) \in S L(2, \mathbf{Z})$; then

$$
M=M_{\infty} \gamma, \quad \text { where } M_{\infty}=\left(\begin{array}{cc}
0 & \delta_{1} \\
0 & \delta_{2}
\end{array}\right), \quad \text { for some } \delta_{1}, \delta_{2} \in N^{-1} \mathbf{Z}-\{0\} .
$$

(In fact, the $i$ th row of $M$ is $\left(\delta_{i} c^{\prime}, \delta_{i} d^{\prime}\right)$, and $\delta_{i}$ is essentially the gcd of the entries of that row.) Using the fact that $\gamma$ normalizes $\Gamma$, we obtain that

$$
\begin{aligned}
\gamma \Gamma_{M} \gamma^{-1} & =\Gamma_{M_{\infty}}=\left\{\left(\begin{array}{cc}
1 & N t \\
0 & 1
\end{array}\right) \mid t \in \mathbf{Z}\right\}, \\
\gamma \mathcal{F}_{M} & =\mathcal{F}_{M_{\infty}}=\{z \mid 0 \leq x<N, y>0\} .
\end{aligned}
$$

It is then a simple matter to conclude from the cuspidality of $f$ that

$$
I_{M, \mathcal{F}_{M}, f}=I_{M_{\infty}, \mathcal{F}_{M_{\infty}},\left.f\right|_{k} \gamma^{-1}}=0 .
$$

This concludes the proof of (2.15). Invoking parts (2) and (3) of Proposition 2.2 now yields (2.16).

We now use the Manin relations between the period symbols in (2.16) to deduce relations between the Eisenstein series. The relations involving $\sigma$ follow easily from the facts that $E_{\ell,-\lambda}=(-1)^{\ell} E_{\ell, \lambda}$ and that powers of $z$ transform nicely under $\sigma$. We state them for the record; they are trivial to see directly (the reader is encouraged to make the connection with the reasoning in Theorem 2.8 or (2.27) below):

$$
E_{\ell^{\prime}+1, \lambda}(z, \bar{s}) E_{m^{\prime}+1, \mu}(z, \bar{s})+(-1)^{\ell^{\prime}} E_{m^{\prime}+1, \mu}(z, \bar{s}) E_{\ell^{\prime}+1,-\lambda}(z, \bar{s})=0 .
$$

The relations involving $\tau$ are the interesting ones. However, if we immediately apply $\tau$ to the powers of $z$ and insist on expanding all polynomials we encounter into linear combinations of powers of $z$, the computation becomes messy. A better way is to use $w$ th powers of linear polynomials; linear combinations of these give all polynomials of degree $\leq w$. The following elementary observation gives the (nice) behavior of such $w$ th powers under $\tau$, and as a side benefit highlights the fact that $\tau$ has order 3 .

Lemma 2.6. Let $a, b, c \in \mathbf{C}$ satisfy $a+b+c=0$. Then

$$
\left.(-a z+b)^{w}\right|_{-w} \tau=(-b z+c)^{w},\left.\quad(-a z+b)^{w}\right|_{-w} \tau^{2}=(-c z+a)^{w} .
$$

The binomial coefficients in the expansion of $(-a z+b)^{w}$ also echo the values of $G_{w, \ell^{\prime}, m^{\prime}}(0)$, especially since we eventually want to evaluate the Eisenstein series at $s=0$. We therefore introduce the following notation for various linear combinations of Eisenstein series. 
Definition 2.7. Fix the value of $w=k-2 \geq 0$. For $a, b \in \mathbf{C}, \lambda, \mu \in N^{-1} \mathbf{Z}^{2}$, and $s \in \mathbf{C}$, define the antiholomorphic function of $a, b, s$ (and "weight $k$ " function of $z \in \mathcal{H}$ ) using the functions $G_{w, \ell^{\prime}, m^{\prime}}$ of (2.17):

$$
L_{\lambda, \mu, a, b}(z, s)=\sum_{\substack{\ell^{\prime}+m^{\prime}=w \\
\ell^{\prime}, m^{\prime} \geq 0}}\left(\begin{array}{l}
w \\
\ell^{\prime}
\end{array}\right)\left(G_{w, \ell^{\prime}, m^{\prime}}(\bar{s})\right)^{-1} \bar{a}^{\ell^{\prime} \bar{b}^{m^{\prime}}} E_{\ell^{\prime}+1, \lambda}(z, \bar{s}) E_{m^{\prime}+1, \mu}(z, \bar{s}) .
$$

The above function is anti-meromorphic in $s$, and in fact is anti-holomorphic for all $s \in \mathbf{C}$ since $\Gamma(s / 2+\ell) E_{\ell, \lambda}(z, s)$ is entire. In particular, we have

$$
L_{\lambda, \mu, a, b}(z, 0)=\sum_{\substack{\ell+m=k \\ \ell, m \geq 1}} \bar{a}^{\ell-1} \bar{b}^{m-1} E_{\ell, \lambda}(z) E_{m, \mu}(z) .
$$

We note here an easy symmetry in $L$ with respect to either exchanging $\lambda$ and $\mu$ or changing their $\operatorname{sign}(\mathrm{s})$ :

$$
L_{\lambda, \mu, a, b}(z, s)=L_{\mu, \lambda, b, a}(z, s)=-L_{-\lambda, \mu,-a, b}(z, s) .
$$

We are ready to state our main result.

Theorem 2.8. Fix $k$ and $w$, as before. Let $\lambda, \mu, \nu \in N^{-1} \mathbf{Z}^{2}$ satisfy $\lambda+\mu+\nu \equiv$ $(0,0) \bmod \mathbf{Z}^{2}$, and let $a, b, c \in \mathbf{C}$ satisfy $a+b+c=0$. Then for all cusp forms $f \in \mathcal{S}_{k}(\Gamma)$, and for all $s$, we have

$$
\left\langle f(z), L_{\lambda, \mu, a, b}(z, s)+L_{\mu, \nu, b, c}(z, s)+L_{\nu, \lambda, c, a}(z, s)\right\rangle_{\Gamma}=0 .
$$

In particular, taking $s=0$, we obtain that the following expression is orthogonal to all cusp forms $f \in \mathcal{S}_{k}(\Gamma)$ :

$$
\begin{aligned}
& \sum_{\substack{\ell+m=k \\
\ell, m \geq 1}} \bar{a}^{\ell-1} \bar{b}^{m-1} E_{\ell, \lambda}(z) E_{m, \mu}(z) \\
+ & \sum_{\substack{\ell+m=k \\
\ell, m \geq 1}} \bar{b}^{\ell-1} \bar{c}^{m-1} E_{\ell, \mu}(z) E_{m, \nu}(z) \\
+ & \sum_{\substack{\ell+m=k \\
\ell, m \geq 1}} \bar{c}^{\ell-1} \bar{a}^{m-1} E_{\ell, \nu}(z) E_{m, \lambda}(z) .
\end{aligned}
$$

Proof. It is enough for us to prove (2.28) when Re $s>2$. By taking linear combinations of (2.16), we obtain the following identity

$$
\begin{aligned}
&\left\langle f(z), L_{\lambda, \mu, a, b}(z, s)\right\rangle_{\Gamma}=i^{w} \pi 2^{-w-s} \times \\
& \times {\left[\sum_{M \in Y_{\lambda, \mu}^{+}}(\operatorname{det} M)^{-s-k / 2}\left[\left.f\right|_{k} M^{-1},(-b z+a)^{w}\right]\right.} \\
&\left.\quad+\sum_{M \in Y_{\mu, \lambda}^{+}}(\operatorname{det} M)^{-s-k / 2}\left[\left.f\right|_{k} M^{-1},(-a z+b)^{w}\right]\right] \\
&=i^{w} \pi 2^{-w-s}\left[S\left(\lambda, \mu,(-b z+a)^{w}, \mathcal{I}\right)+S\left(\mu, \lambda,(-a z+b)^{w}, \mathcal{I}\right)\right] .
\end{aligned}
$$

Here we temporarily introduce the notation

$$
S(\alpha, \beta, P(z), g)=\sum_{M \in Y_{\alpha, \beta}^{+}}(\operatorname{det} M)^{-s-k / 2}\left[\left.f\right|_{k} M^{-1} g, P(z)\right] .
$$


A similar identity to (2.32) holds for the inner products of $f$ with $L_{\mu, \nu, b, c}$ and $L_{\nu, \lambda, c, a}$. All these identities share the common factor $i^{w} \pi 2^{-w-s}$, which we can ignore since our goal is to show that the sum is zero.

The Manin relations (1.7), combined with (2.24), imply that

$$
\begin{aligned}
& S\left(\lambda, \mu,(-b z+a)^{w}, \mathcal{I}\right)+S\left(\mu, \lambda,(-a z+b)^{w}, \mathcal{I}\right) \\
+ & S\left(\lambda, \mu,(-a z+c)^{w}, \tau\right)+S\left(\mu, \lambda,(-b z+c)^{w}, \tau\right) \\
+ & S\left(\lambda, \mu,(-c z+b)^{w}, \tau^{2}\right)+S\left(\mu, \lambda,(-c z+a)^{w}, \tau^{2}\right) \\
& =0 .
\end{aligned}
$$

We now claim that

$$
\begin{aligned}
S\left(\lambda, \mu,(-a z+c)^{w}, \tau\right) & =S\left(\nu, \lambda,(-a z+c)^{w}, \mathcal{I}\right), \\
S\left(\mu, \lambda,(-b z+c)^{w}, \tau\right) & =S\left(\nu, \mu,(-b z+c)^{w}, \mathcal{I}\right), \\
S\left(\lambda, \mu,(-c z+b)^{w}, \tau^{2}\right) & =S\left(\mu, \nu,(-c z+b)^{w}, \mathcal{I}\right), \\
S\left(\mu, \lambda,(-c z+a)^{w}, \tau^{2}\right) & =S\left(\lambda, \nu,(-c z+a)^{w}, \mathcal{I}\right) .
\end{aligned}
$$

To prove this claim, we observe that the first two identities in (2.33) there follow easily from the fact that $M \mapsto \tau^{-1} M$ is a determinant-preserving bijection from $Y_{\alpha, \beta}^{+}$to $Y_{\gamma, \alpha}^{+}$whenever $\{\alpha, \beta, \gamma\}=\{\lambda, \mu, \nu\}$. Similarly, the last two identities hold because $M \mapsto \tau^{-2} M$ is a determinant-preserving bijection from $Y_{\alpha, \beta}^{+}$to $Y_{\beta, \gamma}^{+}$.

The above claim, combined with (2.30) and (2.32), easily implies the desired result (2.28), thereby completing our proof. Note that we "diagonally" combine terms from the second and third lines of (2.32) to obtain the inner products with $L_{\mu, \nu, b, c}$ and $L_{\nu, \lambda, c, a}$.

\section{RELATion to holOMORPHiC EISENSTEIN SERIES}

Our result in Theorem 2.8 says that the expression (2.29) is orthogonal to all cusp forms. Since this is true for arbitrary choices of $a$ and $b$ (with $c=-a-b$ ), we can expand everything into a polynomial in $a$ and $b$, and obtain an expression for the coefficient of each term $a^{i} b^{j}$; each such expression will be orthogonal to $\mathcal{S}_{k}(\Gamma)$.

It is tempting to conclude that these expressions must therefore be Eisenstein series on $\Gamma$. However the non-holomorphic weight 2 Eisenstein series, such as $E_{2, \lambda}$, cause problems. We will therefore take linear combinations, so as to obtain holomorphic modular forms orthogonal to all holomorphic cusp forms; these new expressions will be genuine holomorphic Eisenstein series.

We introduce the following lighter notation for Eisenstein series in this section:

$$
\begin{array}{cl}
A_{\lambda}=E_{1, \lambda}, \quad B_{\lambda}=E_{2, \lambda}, & C_{\lambda}=E_{3, \lambda}, \quad D_{\lambda}=E_{4, \lambda}, \\
Z_{\lambda}=E_{k-1, \lambda}, & Y_{\lambda}=E_{k-2, \lambda} .
\end{array}
$$

We recall that $E_{\ell,-\lambda}=(-1)^{\ell} E_{\ell, \lambda}$, so the expressions $A_{\lambda}$ and $C_{\lambda}$ are odd functions of $\lambda$, and in particular $A_{0}=0=C_{0}$; similarly, $B_{\lambda}$ and $D_{\lambda}$ are even functions of $\lambda$. We also remark that any difference $B_{\lambda}(z)-B_{\mu}(z)$ is a holomorphic function of $z$. In particular, as is classical, $B_{\lambda}-B_{0}$ is equal to the value of the Weierstrass $\wp$-function (see for example (3.5) in [KM12], as well as the proof of Proposition 2.4 in that article):

$$
B_{\lambda}(z)-B_{0}(z)=\wp\left(z_{\lambda} ; \mathbf{Z} z+\mathbf{Z}\right), \quad z_{\lambda}:=\lambda_{1} z+\lambda_{2} .
$$


In the notation of (3.1), Theorem 2.8 then states the following, after replacing $a, b, c$ with their complex conjugates. Recall that $\lambda+\mu+\nu \equiv 0 \bmod \mathbf{Z}^{2}$.

$$
\begin{aligned}
& a^{w} Z_{\lambda} A_{\mu}+a^{w-1} b Y_{\lambda} B_{\mu}+\cdots+a b^{w-1} B_{\lambda} Y_{\mu}+b^{w} A_{\lambda} Z_{\mu} \\
+ & b^{w} Z_{\mu} A_{\nu}+b^{w-1}(-a-b) Y_{\mu} B_{\nu}+\cdots+b(-a-b)^{w-1} B_{\mu} Y_{\nu}+(-a-b)^{w} A_{\mu} Z_{\nu} \\
+ & (-a-b)^{w} Z_{\nu} A_{\lambda}+(-a-b)^{w-1} a Y_{\nu} B_{\lambda}+\cdots+(-a-b) a^{w-1} B_{\nu} Y_{\lambda}+a^{w} A_{\nu} Z_{\lambda} \\
& \in \mathcal{S}_{k}(\Gamma)^{\perp} .
\end{aligned}
$$

Before discussing the general situation, we work out the identities for some small weights directly, and relate them to elliptic functions.

Weight $k=2$ : Here $w=0$ and we have $A_{\lambda} A_{\mu}+A_{\mu} A_{\nu}+A_{\nu} A_{\lambda} \in \mathcal{S}_{2}^{\perp}$. This expression is already holomorphic, and is hence a holomorphic Eisenstein series of weight 2. This result appears in Borisov-Gunnells (Propositions 3.7 and 3.8 of [BG01b]) and is reproved as equation (4.10) of [KM12]. An important special case is when $\mu=0$ and $\nu=-\lambda$. We then have $A_{\mu}=0$ and $A_{\nu}=-A_{\lambda}$, and conclude that $A_{\lambda}^{2}$ is a holomorphic Eisenstein series (actually, this fact was used in proving the more general result in BG01b and KM12]). Combining these, we see that under our standing assumption $\lambda+\mu+\nu \equiv 0 \bmod \mathbf{Z}^{2}$, the expression $\left(A_{\lambda}+A_{\mu}+A_{\nu}\right)^{2}$ is a holomorphic weight 2 Eisenstein series. Let us assume that moreover $\lambda, \mu, \nu$ are all nonzero, and choose specific representatives modulo $\mathbf{Z}^{2}$ for which $\lambda+\mu+\nu=0$. Let $\zeta$ and $\wp$ denote the standard Weierstrass elliptic functions with respect to the lattice $\mathbf{Z} z+\mathbf{Z}$; then our assertion about $\left(A_{\lambda}+A_{\mu}+A_{\nu}\right)^{2}$ is in fact a consequence of the classical identity $\left(\zeta\left(z_{\lambda}\right)+\zeta\left(z_{\mu}\right)+\zeta\left(z_{\nu}\right)\right)^{2}=\wp\left(z_{\lambda}\right)+\wp\left(z_{\mu}\right)+\wp\left(z_{\nu}\right)$. For a recent treatment, see for example equation (3.8) and Corollary 3.13 of [KM12, as well as the treatment in Paş06.

Weight $k=3$ : When $w=1$, we obtain from (3.3) that $a B_{\lambda} A_{\mu}+b A_{\lambda} B_{\mu}+$ $b B_{\mu} A_{\nu}+(-a-b) A_{\mu} B_{\nu}+(-a-b) B_{\nu} A_{\lambda}+a A_{\nu} B_{\lambda} \in \mathcal{S}_{3}^{\perp}$. The coefficient of $a$ says that $B_{\lambda} A_{\mu}-A_{\mu} B_{\nu}-B_{\nu} A_{\lambda}+A_{\nu} B_{\lambda} \in \mathcal{S}_{3}^{\perp}$. The coefficient of $b$ gives an equivalent identity upon exchanging the roles of $\lambda$ and $\mu$. We also note the identity arising from the special case $\mu=0, \nu=-\lambda$, which implies that $A_{\lambda} B_{\lambda} \in \mathcal{S}_{3}^{\perp}$. (The other case, $\mu=-\lambda, \nu=0$, also implies this fact, since the terms $\pm A_{\lambda} B_{0}$ cancel; this phenomenon is specific to weight 3.) Thus any nonholomorphic product $A_{\lambda} B_{\mu}$ is congruent modulo $\mathcal{S}_{3}^{\perp}$ to the holomorphic modular form $A_{\lambda}\left(B_{\mu}-B_{\lambda}\right)$. Combining this with our previous assertion implies that $\left(A_{\lambda}+A_{\mu}+A_{\nu}\right)\left(B_{\lambda}-B_{\nu}\right)$ is a holomorphic weight 3 Eisenstein series; it is in fact equal to $C_{\lambda}-C_{\nu}$. In the context of our remark for weight 2 , this is the classical identity

$$
\left(\zeta\left(z_{\lambda}\right)+\zeta\left(z_{\mu}\right)+\zeta\left(z_{\nu}\right)\right)\left(\wp\left(z_{\lambda}\right)-\wp\left(z_{\mu}\right)\right)=-2^{-1}\left(\wp^{\prime}\left(z_{\lambda}\right)-\wp^{\prime}\left(z_{\mu}\right)\right) .
$$

See also [KM12], Introduction and equation (4.39).

Weight $k=4$ : The coefficient of $a^{2}$ in (3.3) shows that $C_{\lambda} A_{\mu}+A_{\mu} C_{\nu}+C_{\nu} A_{\lambda}-$ $B_{\nu} B_{\lambda}+A_{\nu} C_{\lambda} \in \mathcal{S}_{4}^{\perp}$; the coefficient of $b^{2}$ yields the same, upon exchanging $\lambda$ and $\mu$. Moreover, the coefficient of $a b$ is $B_{\lambda} B_{\mu}-B_{\mu} B_{\nu}+2 A_{\mu} C_{\nu}+2 C_{\nu} A_{\lambda}-B_{\nu} B_{\lambda} \in \mathcal{S}_{4}^{\perp}$. By taking the special case $\mu=0, \nu=-\lambda$, we deduce from either assertion above that $2 A_{\lambda} C_{\lambda}+B_{\lambda}^{2} \in \mathcal{S}_{4}^{\perp}$ (once again, terms with $B_{0}$ cancel). On the other hand, the special case $\nu=0, \mu=-\lambda$ yields $A_{\lambda} C_{\lambda}+B_{0} B_{\lambda} \in \mathcal{S}_{4}^{\perp}$ and $B_{\lambda}^{2}-2 B_{\lambda} B_{0} \in \mathcal{S}_{4}^{\perp}$. (This last fact can alternatively be deduced from the previously computed elements of $\mathcal{S}_{4}^{\perp}$. Note also that specializing to $\lambda=0, \nu=-\mu$ yields no new elements of $\mathcal{S}_{4}^{\perp}$.) We deduce in particular that $B_{0}^{2} \equiv A_{0} C_{0} \equiv 0 \bmod \mathcal{S}_{4}^{\perp}$; this can also be seen because 
$B_{0}^{2}$ is invariant under the full group $S L_{2}(\mathbf{Z})$, whence the inner product of $B_{0}^{2}$ with any cusp form $f$ on $\Gamma$ is essentially the inner product with the $\operatorname{trace} \operatorname{tr}_{S L(2, \mathbf{Z})}^{\Gamma} f \in$ $\mathcal{S}_{4}(S L(2, \mathbf{Z}))=0$. It follows that $\left(B_{\lambda}-B_{0}\right)^{2}=B_{\lambda}^{2}-2 B_{\lambda} B_{0}+B_{0}^{2} \in \mathcal{S}_{4}^{\perp}$, a fact that can be seen directly from the classical identity $6 D_{\lambda}=\wp^{\prime \prime}\left(z_{\lambda}\right)=6 \wp\left(z_{\lambda}\right)^{2}-30 D_{0}$.

To wrap up the case of weight $k=4$, we construct holomorphic modular forms that are congruent (modulo $\mathcal{S}_{4}^{\perp}$ ) to the nonholomorphic products of the form $B_{\lambda} B_{\mu}$. One way to do this is to write $B_{\lambda} B_{\mu}=\left(B_{\lambda}-B_{0}\right)\left(B_{\mu}-B_{0}\right)+B_{\lambda} B_{0}+B_{\mu} B_{0}-B_{0}^{2} \equiv$ $\left(B_{\lambda}-B_{0}\right)\left(B_{\mu}-B_{0}\right)-A_{\lambda} C_{\lambda}-A_{\mu} C_{\mu}(+0)$. Using this congruence, we obtain from the coefficients of $a^{2}$ and $a b$ in the previous paragraph the statement that the following two expressions are holomorphic Eisenstein series of weight 4: $\left(A_{\lambda}+A_{\mu}+A_{\nu}\right)\left(C_{\lambda}+\right.$ $\left.C_{\nu}\right)-\left(B_{\lambda}-B_{0}\right)\left(B_{\nu}-B_{0}\right)$ and $\left(B_{\lambda}-B_{0}\right)\left(B_{\mu}-B_{0}\right)-\left(B_{\lambda}-B_{0}\right)\left(B_{\nu}-B_{0}\right)-\left(B_{\mu}-\right.$ $\left.B_{0}\right)\left(B_{\nu}-B_{0}\right)+2\left(A_{\lambda}+A_{\mu}+A_{\nu}\right) C_{\nu}$. We leave it to the reader to verify that it would have sufficed to prove that either one of the above expressions was an Eisenstein series (for all permutations of $(\lambda, \mu, \nu))$ in order to deduce the same about the other expression.

The situation for general weight $k$ : Here we shall content ourselves with showing that every potentially nonholomorphic term in (3.3), such as $Y_{\lambda} B_{\mu}$, can be modified by an appropriate element of $\mathcal{S}_{k}^{\perp}$ to obtain a holomorphic form. To do this, consider the coefficient of $a^{w}$ in (3.3) in the special case $\mu=0, \nu=-\lambda$. This coefficient is

$$
\begin{aligned}
Z_{\lambda} A_{0} & +(-1)^{w} A_{0} Z_{-\lambda}[\text { both these terms are zero }] \\
& +(-1)^{w} Z_{-\lambda} A_{\lambda}+(-1)^{w-1} Y_{-\lambda} B_{\lambda}+\cdots+(-1) B_{-\lambda} Y_{\lambda}+A_{-\lambda} Z_{\lambda} \\
=-\left(Z_{\lambda} A_{\lambda}\right. & \left.+Y_{\lambda} B_{\lambda}+\cdots+B_{\lambda} Y_{\lambda}+A_{\lambda} Z_{\lambda}\right) \in \mathcal{S}_{k}^{\perp} .
\end{aligned}
$$

After dividing by -2 , we obtain that $B_{\lambda} Y_{\lambda}$ is congruent modulo $\mathcal{S}_{k}^{\perp}$ to a holomorphic expression in terms of the other products such as $A_{\lambda} Z_{\lambda}$. This allows us to rewrite any general product such as $Y_{\lambda} B_{\mu}$ as $Y_{\lambda}\left(B_{\mu}-B_{\lambda}\right)+B_{\lambda} Y_{\lambda} \equiv Y_{\lambda}\left(B_{\mu}-B_{\lambda}\right)+$ something holomorphic. The careful reader will note that our discussion above seems to be restricted to $k \geq 5$, because we implicitly assumed that the terms $Y_{\lambda} B_{\lambda}$ and $B_{\lambda} Y_{\lambda}$ were distinct; moreover, when $k=4$, the $Y$ s are actually $B \mathrm{~s}$, and $B_{\lambda}\left(B_{\mu}-B_{\lambda}\right)$ is not holomorphic. However, it turns out upon more careful investigation that the above technique still works for $2 \leq k \leq 4$, and in any case, we have already written down explicit formulas for these small weights.

The cleanest way to find holomorphic relations in all weights is to do so on the level of (3.3), instead of teasing out each coefficient of $a^{i} b^{j}$ separately. The result is even better expressed on the level of a generating series, in which setting it becomes a different proof of the main result in Paş06 (see most notably the top of p. 16). We shall go back to writing the result in terms of $a, b, c$ where $a+b+c=0$, instead of substituting $c=-a-b$.

Theorem 3.1. Take $k, w, \lambda, \mu, \nu, a, b, c$, be as in Theorem 2.8. Then the following expression is a holomorphic Eisenstein series of weight $k$ :

$$
\begin{aligned}
& \left(A_{\lambda}+A_{\mu}+A_{\nu}\right)\left(a^{w} Z_{\lambda}+b^{w} Z_{\mu}+c^{w} Z_{\nu}\right) \\
+ & \left(a B_{\lambda}+b B_{\mu}+c B_{\nu}\right)\left(a^{w-1} Y_{\lambda}+b^{w-1} Y_{\mu}+c^{w-1} Y_{\nu}\right) \\
+ & \cdots \\
+ & \left(a^{w-1} Y_{\lambda}+b^{w-1} Y_{\mu}+c^{w-1} Y_{\nu}\right)\left(a B_{\lambda}+b B_{\mu}+c B_{\nu}\right) \\
+ & \left(a^{w} Z_{\lambda}+b^{w} Z_{\mu}+c^{w} Z_{\nu}\right)\left(A_{\lambda}+A_{\mu}+A_{\nu}\right) .
\end{aligned}
$$


Assume furthermore that $|a|,|b|,|c|$ are sufficiently small. Define power series

$$
F_{\lambda}(a)=A_{\lambda}+a B_{\lambda}+a^{2} C_{\lambda}+\cdots, \quad \text { similarly for } F_{\mu}(b), F_{\nu}(c) .
$$

Then (3.6) says that the power series expansion of $\left(F_{\lambda}(a)+F_{\mu}(b)+F_{\nu}(c)\right)^{2}$ in a neighborhood of zero in the hyperplane $a+b+c=0$ has as its degree $w$ term a holomorphic Eisenstein series of weight $w+2$. In the special case when $\lambda, \mu, \nu$ are all nonzero modulo $\mathbf{Z}^{2}$ and satisfy $\lambda+\mu+\nu=0$ (not just congruence modulo $\mathbf{Z}^{2}$ ), we can see this from the fact ([Paş06, Theorem 3.1 and Observation 4.2.5) that

$$
\begin{aligned}
F_{\lambda}(a)+F_{\mu}(b)+F_{\nu}(c) & =\zeta\left(z_{\lambda}-a\right)+\zeta\left(z_{\mu}-b\right)+\zeta\left(z_{\nu}-c\right), \\
\left(F_{\lambda}(a)+F_{\mu}(b)+F_{\nu}(c)\right)^{2} & =\wp\left(z_{\lambda}-a\right)+\wp\left(z_{\mu}-b\right)+\wp\left(z_{\nu}-c\right) \\
=B_{\lambda}+B_{\mu}+B_{\nu} & -3 B_{0}+2\left(a C_{\lambda}+b C_{\mu}+c C_{\nu}\right) \\
+3\left(a^{2} D_{\lambda}\right. & \left.+b^{2} D_{\mu}+c^{2} D_{\nu}\right)+\cdots
\end{aligned}
$$

In other words, in this special case, the expression in (3.6) is equal to the holomorphic Eisenstein series $(w+1)\left(a^{w} E_{w+2, \lambda}+b^{w} E_{w+2, \mu}+c^{w} E_{w+2, \nu}\right)$ when $w \geq 1$. For the case $w=0$, the value is $E_{2, \lambda}+E_{2, \mu}+E_{2, \nu}-3 E_{2,0}$.

Remark 3.2. The case where one or three of $\lambda, \mu, \nu$ are zero modulo $\mathbf{Z}^{2}$ is also proved in Paş06, Proof of Theorem 0.1 (pp. 15-17), and one can obtain in principle explicit values for the expression of (3.6) for those cases as well.

Proof of Theorem [3.1. We originally obtained the first assertion as the result of taking twice the expression in (3.3) and replacing each possibly nonholomorphic term such as $Y_{\lambda} B_{\mu}$ by something holomorphic congruent to it modulo $\mathcal{S}_{k}^{\perp}$, as described in the discussion following (3.5). But now that we have found the expression (3.6), the easiest way to show that it is orthogonal to all cusp forms is to expand each line of (3.6) into nine terms, which we index by the ordered pairs $\{(\lambda, \lambda),(\lambda, \mu),(\lambda, \nu),(\mu, \lambda), \ldots,(\nu, \nu)\}$. The sum of all terms corresponding to $(\mu, \lambda),(\nu, \mu)$, and $(\lambda, \nu)$ is equal to the expression in (3.3), which belongs to $\mathcal{S}_{k}^{\perp}$. Similarly, the terms corresponding to $(\lambda, \mu),(\mu, \nu)$, and $(\nu, \lambda)$ add up to a rearrangement of (3.3) (read each row of (3.3) from right to left). We are left with the sums of terms corresponding to each of $(\lambda, \lambda),(\mu, \mu)$, and $(\nu, \nu)$, each of which yields a multiple of an expression like (3.5). Thus we obtain that (3.6) is orthogonal to all holomorphic cusp forms. To see that it is holomorphic (and hence an Eisenstein series), it suffices to observe that the only potentially nonholomorphic part of (3.6) comes from the expression

$$
a B_{\lambda}+b B_{\mu}+c B_{\nu}=a B_{\lambda}+b B_{\mu}+(-a-b) B_{\nu}=a\left(B_{\lambda}-B_{\nu}\right)+b\left(B_{\mu}-B_{\nu}\right)
$$

which is holomorphic, after all.

As for the second assertion, namely (3.8), it boils down in light of (3.4) to verifying (i) the series expansion in terms of $(a, b, c)$ near 0 of the holomorphic function $\zeta\left(z_{\lambda}-a\right)+\zeta\left(z_{\mu}-b\right)+\zeta\left(z_{\nu}-c\right)$, and (ii) the holomorphic series expansion $\wp\left(z_{\lambda}-a\right)=B_{\lambda}-B_{0}+2 a C_{\lambda}+3 a^{2} D_{\lambda}+\cdots$. The expansion (ii) is a straightforward application of Taylor's theorem; since $\zeta^{\prime}=\wp$, we deduce (i), possibly up to a constant, by integration. It remains to observe that $A_{\lambda}+A_{\mu}+A_{\nu}=\zeta\left(z_{\lambda}\right)+$ $\zeta\left(z_{\mu}\right)+\zeta\left(z_{\nu}\right)$, which follows from Corollary 3.13 of KM12. 


\section{Partial Results on Hecke operators}

In this final section, we prove a result that is related to taking the trace of a product $E_{\ell, \lambda} E_{m, \mu}$ of two Eisenstein series from a higher level $\Gamma(N M)$ to a lower level $\Gamma(M)$. This is also related to the question of how Hecke operators act on a product of two Eisenstein series. The results we obtain are somewhat indirect, but of interest; they generalize both the statement and proof of Proposition 4.6 and the first half of Proposition 4.11 in KM12, which basically deal with the cases $\ell=1$ and $m \in\{1,2\}$ of the general result in this section.

As mentioned in [KM12, most particularly Proposition 4.6 there, a key case of such a trace is to be able to express the sum

$$
\sum_{\tau \in N^{-1} \mathbf{Z}^{2} / \mathbf{Z}^{2}} E_{\ell, \lambda+\tau} E_{m, \mu-S \tau}
$$

as a suitable linear combination of products of Eisenstein series. Here $\lambda, \mu \in$ $M^{-1} \mathbf{Z}^{2}$, and $S \in \mathbf{Z}$; without loss of generality, $0 \leq S<N$. The expression $\tau \in$ $N^{-1} \mathbf{Z}^{2} / \mathbf{Z}^{2}$ means that $\tau=\left(\tau_{1}, \tau_{2}\right)$ ranges over any set of representatives of these cosets: for example, $\tau_{1}, \tau_{2}$ can vary independently in $\{0,1 / N, 2 / N, \ldots,(N-1) / N\}$. Thus our notation $(\lambda, \mu, \tau, N, M, S)$ here corresponds to $(A, B, T, n, \ell, s)$ in Proposition 4.6 of [KM12]. Note that each individual term $E_{\ell, \lambda+\tau} E_{m, \mu-S \tau}$ can have level up to $M N$, but the sum transforms according to $\Gamma(M)$. Our main result is that the sum in (4.1) can be written (up to elements orthogonal to cusp forms) in terms of a linear combination of products of two Eisenstein series on the lower level $M$, each product being of the form $E_{\hat{\ell}, p \lambda+q \mu} E_{\hat{m}, r \lambda+s \mu}$, with $(p, q, r, s)$ taking the role of $(a, b, c, d)$ from our earlier article.

It turns out to be simpler to state the result using the linear combinations $L_{\lambda, \mu, a, b}(z, s)$ from (2.25), where $a, b \in \mathbf{C}$ are arbitrary parameters; then, taking the coefficient of $\bar{a}^{\ell-1} \bar{b}^{m-1}$ in any such expression below gives us the value of (4.1).

Proposition 4.1. Fix $k \geq 2$ and $w=k-2$ as usual. Let $(\lambda, \mu, \tau, N, M, S)$ be as in the preceding discussion. Then there exist finitely many matrices $\left(\begin{array}{ccc}p_{i} & q_{i} \\ r_{i} & s_{i}\end{array}\right) \in$ $M_{2}(\mathbf{Z})$ and constants $c_{i} \in \mathbf{Z}$, all depending only on $N$ and $S$ but not on any other parameters, such that we have a congruence modulo $\mathcal{S}_{k}^{\perp}$ of the form:

$$
\sum_{\tau \in N^{-1} \mathbf{Z}^{2} / \mathbf{Z}^{2}} L_{\lambda+\tau, \mu-S \tau, a, b} \equiv N^{\bar{s}+1} \sum_{i} c_{i} L_{p_{i} \lambda+q_{i} \mu, r_{i} \lambda+s_{i} \mu, p_{i} a+q_{i} b, r_{i} a+s_{i} b} .
$$

Here $s$ is the complex parameter in the Eisenstein series; we mainly care about the case $s=0$. The matrices $\left(\begin{array}{cc}p_{i} & q_{i} \\ r_{i} & s_{i}\end{array}\right)$ all satisfy

$$
\operatorname{det}\left(\begin{array}{cc}
p_{i} & q_{i} \\
r_{i} & s_{i}
\end{array}\right)=N, \quad \quad p_{i}-S q_{i} \equiv r_{i}-S s_{i} \equiv 0 \quad(\bmod N) .
$$

Proof. In the above sum, the value of $S$ matters only modulo $N$, so, as already noted, we may assume that $0 \leq S<N$. The proof is then by induction on $S$, and follows closely the technique of Proposition 4.6 in KM12. The base case $S=0$ is easy to dispose of, since the definition of Eisenstein series immediately implies that $\sum_{\tau \in N^{-1} \mathbf{Z}^{2} / \mathbf{Z}^{2}} E_{\ell, \lambda+\tau}(z, \bar{s})=N^{\ell+\bar{s}} E_{\ell, N \lambda}(z, \bar{s})$. This implies that

$$
\sum_{\tau \in N^{-1} \mathbf{Z}^{2} / \mathbf{Z}^{2}} L_{\lambda+\tau, \mu, a, b}(z, s)=N^{\bar{s}+1} L_{N \lambda, \mu, N a, b}(z, s) .
$$

So here there is only one matrix, $\left(\begin{array}{cc}p & q \\ r & s\end{array}\right)=\left(\begin{array}{cc}N & 0 \\ 0 & 1\end{array}\right)$. 
The next case, $S=1$, uses the relation $L_{\lambda+\tau, \mu-\tau, a, b}(z, s)+L_{\mu-\tau,-\lambda-\mu, b,-a-b}(z, s)+$ $L_{-\lambda-\mu, \lambda+\tau,-a-b, a}(z, s) \equiv 0\left(\bmod \mathcal{S}_{k}^{\perp}\right)$, which is essentially (2.28). Adding up over all $\tau$, and using (4.4), we obtain

$$
\sum_{\tau \in N^{-1} \mathbf{Z}^{2} / \mathbf{Z}^{2}} L_{\lambda+\tau, \mu-\tau, a, b} \equiv N^{\bar{s}+1}\left(-L_{N \mu,-\lambda-\mu, N b,-a-b}-L_{-\lambda-\mu, N \lambda,-a-b, N a}\right) .
$$

Thus the matrices are $\left(\begin{array}{cc}p_{i} & q_{i} \\ r_{i} & S_{i}\end{array}\right)=\left(\begin{array}{cc}0 & N \\ -1 & -1\end{array}\right),\left(\begin{array}{cc}-1 & -1 \\ N & 0\end{array}\right)$. Note that the second term above is the result of combining (4.4) with (2.27).

For larger $S$, we transform the sum involving $S \tau$ into one involving just $\tau$, at the expense of introducing a sum over $\sigma \in S^{-1} \mathbf{Z}^{2} / \mathbf{Z}^{2}$; this uses (4.4), with $S$ instead of $N$, as follows. (Using $-\sigma$ instead of $\sigma$ is more convenient for later.)

$$
\sum_{\tau \in N^{-1} \mathbf{Z}^{2} / \mathbf{Z}^{2}} L_{\lambda+\tau, \mu-S \tau, a, b}=\sum_{\tau \in N^{-1} \mathbf{Z}^{2} / \mathbf{Z}^{2}} S^{-\bar{s}-1} \sum_{\sigma \in S^{-1} \mathbf{Z}^{2} / \mathbf{Z}^{2}} L_{\lambda+\tau, \mu / S-\tau-\sigma, a, b / S} .
$$

At this point, we can carry out the sum over $\tau$ first, using (4.5). The result is congruent $\left(\bmod \mathcal{S}_{k}^{\perp}\right)$ to

$$
(N / S)^{\bar{s}+1} \sum_{\sigma \in S^{-1} \mathbf{Z}^{2} / \mathbf{Z}^{2}}\left(-L_{N \mu / S-N \sigma,-\lambda-\mu / S+\sigma, N b / S,-a-b / S}-L_{-\lambda-\mu / S+\sigma, N \lambda,-a-b / S, N a}\right) .
$$

We first address the sum over $\sigma$ of the second $L$ in the above expression. Taking into account the factor $(N / S)^{\bar{s}+1}$, this yields $-N^{\bar{s}+1} L_{-S \lambda-\mu, N \lambda,-S a-b, N a}$; the corresponding matrix is $\left(\begin{array}{cc}p & q \\ r & s\end{array}\right)=\left(\begin{array}{cc}-S & -1 \\ N & 0\end{array}\right)$. We next deal with the sum over $\sigma$ of the first $L$ in (4.7). This sum is of the same type as our original sum over $\tau$, but with the roles of $N$ and $S$ reversed. However, the term $N \sigma$ can be replaced by $N^{\prime} \sigma$, where $N^{\prime}=N \bmod S$. Since $N^{\prime}<S$, we can use induction to conclude that this last sum, combined with the external factor $(N / S)^{\bar{s}+1}$, is congruent $\left(\bmod \mathcal{S}_{k}^{\perp}\right)$ to an expression of the form

$$
(N / S)^{\bar{s}+1} \cdot S^{\bar{s}+1} \sum_{i} c_{i} L_{\hat{p}_{i} \hat{\lambda}+\hat{q}_{i} \hat{\mu}, \hat{r}_{i} \hat{\lambda}+\hat{s}_{i} \hat{\mu}, \hat{p}_{i}(N b / S)+\hat{q}_{i}(-a-b / S), \hat{r}_{i}(N b / S)+\hat{s}_{i}(-a-b / S)},
$$

where $\hat{\lambda}=N \mu / S$ and $\hat{\mu}=-\lambda-\mu / S$; moreover, for each $i$ in the sum, we have $\operatorname{det}\left(\begin{array}{cc}\hat{p}_{i} & \hat{q}_{i} \\ \hat{r}_{i} & \hat{s}_{i}\end{array}\right)=S$ and $-N^{\prime} \hat{p}_{i}+\hat{q}_{i} \equiv-N^{\prime} \hat{r}_{i}+\hat{s}_{i} \equiv 0(\bmod S)$. Now each term above in the sum over $i$ can be rewritten in terms of the original $\lambda, \mu$ as $L_{p_{i} \lambda+q_{i} \mu, r_{i} \lambda+s_{i} \mu, p_{i} a+q_{i} b, r_{i} a+s_{i} b}$, where $\left(\begin{array}{cc}p_{i} & q_{i} \\ r_{i} & s_{i}\end{array}\right)=\left(\begin{array}{cc}-\hat{q}_{i} & \left(\hat{p}_{i} N-\hat{q}_{i}\right) / S \\ -\hat{s}_{i} & \left(\hat{r}_{i} N-\hat{s}_{i}\right) / S\end{array}\right)$. We leave it to the reader to check that these matrices are integral and that they satisfy (4.3). Our proof by induction is now complete.

Corollary 4.2. Consider a sum as in (4.1), except that if $\ell=2$ or $m=2$ we replace every nonholomorphic expression such as $E_{2, \lambda+\tau}$, wherever it appears, with the holomorphic expression $\tilde{E}_{2, \lambda+\tau}=E_{2, \lambda+\tau}-E_{2,0}$. Then the resulting sum is a holomorphic modular form, and is congruent (modulo holomorphic Eisenstein series) to a linear combination of products of two holomorphic Eisenstein series of level $M$.

Proof. Write each $\tilde{E}_{2}$ as a difference of two $E_{2}$ 's, and take the sum over $\tau$ of each product using the result of Proposition 4.1. (Sums such as $\sum_{\tau} E_{2, \lambda+\tau} E_{2,0}$ or $\sum_{\tau} E_{2,0} E_{m, \mu-S \tau}$, which only involve $\tau$ in one factor, are easy to simplify.) This 
involves taking coefficients of some monomials like $\bar{a}^{\ell} \bar{b}^{m}$ in the result of the previous proposition. This shows that the final answer, which is holomorphic, is congruent modulo $\mathcal{S}_{k}^{\perp}$ to some linear combination of products of pairs of (possibly nonholomorphic, if weight 2) Eisenstein series of level $M$. But now modify the result by suitable combinations of expressions like (3.5) to obtain a new holomorphic expression, expressed in terms of level $M$, that is congruent to our desired sum modulo $\mathcal{S}_{k}^{\perp}$. Then the difference between our original desired sum and the new holomorphic expression is both holomorphic and orthogonal to cusp forms, so must be in the desired Eisenstein space of level $M$.

We conclude this article by raising the question of whether one can also generalize the proofs of Proposition 4.8 and the second half of Proposition 4.11 in KM12; these results show that more general traces of products of Eisenstein series, not just those in (4.1), can again be written as combinations of such products at lower level. Generalizing our earlier proofs appears to require a better understanding of the effect of a certain "Fourier transform" on spaces of Eisenstein series, along the lines of Proposition 4.3 of that article, alongside a similar theory of relations between products of Eisenstein series in the image of this Fourier transform. With our current state of knowledge, the stronger results in Section 4 of KM12 can be proved directly only for weights 2 and 3 , as is done in that article. The reason for this is that any product of two Eisenstein series in such low weight must include one Eisenstein series of weight 1; this saves us in our earlier article, because the Fourier symmetry on weight 1 Eisenstein series is essentially the identity transform.

\section{REFERENCES}

[BG01a] Lev A. Borisov and Paul E. Gunnells, Toric varieties and modular forms, Invent. Math. 144 (2001), no. 2, 297-325. MR 1826373 (2002g:11053)

[BG01b] , Toric modular forms and nonvanishing of L-functions, J. Reine Angew. Math. 539 (2001), 149-165. MR 1863857 (2002h:11042)

[BG03] - Toric modular forms of higher weight, J. Reine Angew. Math. 560 (2003), 43-64. MR 1992801 (2004f:11037)

[KM12] Kamal Khuri-Makdisi, Moduli interpretation of Eisenstein series, Int. J. Number Theory 8 (2012), no. 3, 715-748. MR 2904927

[KZ84] W. Kohnen and D. Zagier, Modular forms with rational periods, Modular forms (Durham, 1983), Ellis Horwood Ser. Math. Appl.: Statist. Oper. Res., Horwood, Chichester, 1984, pp. 197-249. MR 803368 (87h:11043)

[Mer94] Loïc Merel, Universal Fourier expansions of modular forms, On Artin's conjecture for odd 2-dimensional representations, Lecture Notes in Math., vol. 1585, Springer, Berlin, 1994, pp. 59-94. MR 1322319 (96h:11032)

[Paş06] Vicenţiu Paşol, A modular symbol with values in cusp forms, http://arxiv.org/abs/math/0611704v1 preprint, 2006.

[Shi76] Goro Shimura, The special values of the zeta functions associated with cusp forms, Comm. Pure Appl. Math. 29 (1976), no. 6, 783-804. MR 0434962 (55 \#7925)

[Shi07] _ Elementary Dirichlet series and modular forms, Springer Monographs in Mathematics, Springer, New York, 2007. MR 2341272 (2008g:11001)

Mathematics Department, American University of Beirut, Bliss Street, Beirut, Lebanon

E-mail address: kmakdisi@aub.edu.lb

Mathematics Department, American University of Beirut, Bliss Street, Beirut, Lebanon

E-mail address: wr07@aub.edu.1b 